\section{LECTURE NOTE VOLUMES}

Brandeis Summer Institute in Theoretical Physics

Vol. 1: Lectures on Strong and Electromagnetic Interactions. By P. T. Matthews, D. R. Yennie and M. E. Mayer. Pp. iii + 343. 24s. Vol. 2: Lectures on Astrophysies and Weak Interactions. By S. Hayakawa, H. Y. Chiu, G. Feinberg and M. Dresden. Pp. v +471. 32s. $6 d$. (Waltham, Mass.: Brandeis University. Distributed by Gordon and Breach, New York, and Blackie and Son, London, 1964.)

T the quickly developing areas of science, such as those to which Lectures on Strong and Electromagnetic Interactions and Lectures on Astrophysics and Weak Interactions offer contributions, the pressure for shorter and shorter publication times for more and more material has extended from the research journals to the text-books; hence the spate of 'lecture note volumes', as they are called. Notes of lectures at the various summer schools are written up either by the lecturer himself, or more commonly by a student attending the school, and published as quickly as possible, usually in paper-back and usually with a minimal amount of editing. Their purpose must be considered primarily didactic. As the editor of the volumes reviewed here claims, "These notes should provide a valuable introduction to areas of contemporary research interest".

A "valuable introduction" for whom? All three articles of Volume 1 would make little sense to a reader without at least some familiarity with the ideas of elementary quantum field theory. Given this background, the notes of the lectures by Matthews provide a readable introduction to the $S$-matrix and its uses in strong interaction physics. There are also useful sections on applications to low-energy $\pi-N$ and $\bar{K}-N$ scattering, the determination of the quantum numbers of resonances and high-energy scattering. I am not so confident of the usefulness of the notes of the lectures by Meyer, which purport to be an introduction to the $S U_{3}$ symmetry scheme of strongly interacting particles. The lectures seem to have been a review very much for the experts; terms such as "Weyl's branching theorem", "Casimir operator", "Okubo's form for the mass matrix" are bandied about without being adequately explained. More readable introductions to the $S U_{3}$ scheme now exist in print which cover essentially the same material. Yennie's article is perhaps more specialized and detailed than the other two articles of Volume 1, but certainly no less readable, nor requiring any more background knowledge. It gives an exposition of the techniques associated with the author's name for making the Lamb shift calculations, and an account of the treatment of the infra-red divergence problem and radiative corrections to high-energy scattering.

There are even more variations in the assumed background knowledge of the readers of the various contributions to Volume 2. The title of the lecture notes of Chiu, "Neutrino Astrophysics", is a little misleading. They are divided into two parts and only the second deals at all with neutrino processes, stellar collapse and neutron stars. I can recommend the first part as a very readable brief introduction to the ideas of astrophysicists on stellar sizes, stellar structure, nuclear reactions in stars and stcllar evolution. Much of the material requires as background no more than an honours degree course in physics. The other astrophysics article, by Hayakawa on "The Origin of Cosmic Rays", precedes that of Chiu in the volume. However, it would not be intelligible to anyone who has not already a fair familiarity with the ideas presented in Chiu's article. The other two articles in Volume 2 are for specialists in elementary particle physics. That of Feinberg is largely on the intermodiate vector boson theory of weak interaction processes, its field theoretic formulation, and possible effects of higher order graphs. The article of Dresden is a very complete and carefully written account of theoretical speculations on the possible existence of particles obeying neither Bose-Einstein nor Fermi-Dirac statistics.

One might well question the wisdom of the continuing publication of these lecture note volumes. They are not books. For, in the interests of speed, there is no attempt on the part of the editor to make uniformity either in lay-out or notation or even in method of referencing. In the second volume even the ordering of the articles seems inappropriate. In all but two cases, the lecturers did not even write their own contributions. In the foreword the editor points out that two of the lecturers "generously devoted time to reviewing the work of their notetakers, but have not undertaken extensive revisions". It seems to me a question not of the generosity of the lecturer but of his responsibility to ensure that whatever is published under his name is as accurately as possible what he wants to say. The editor says that "the notes are intended to be informal presentations, not formal monographs". But the informality of a presentation certainly does not add to its clarity, if it entails, as it seems so often to do in these volumes, undefined notation, carelessly stated principles and badly labelled diagrams. In spite of it all, some things come through and some of the articles will be useful to some people.

J. E. Paton

\section{SPECIAL CERAMICS}

\section{Special Ceramics 1964}

Edited by P. Popper. (Proceedings of a Symposium held by the British Ceramic Research Association.) Pp. xii + 341. (London: Academic Press, Inc. (London), Ltd.; New York: Academic Press, Inc., 1965. Published for the British Ceramic Research Association.) $90 s$.

THE term ceramics, be they special or not, includes a vast range of solid materials. In the past few years the definition, which originally included only materials based on clay, has been widened progressively so that we now readily accept such statements as "In materials there exist some that are neither metals nor elements nor polymers, these are ceramics". Special Ceramics 1964 indicates that even this is not wide enough and 'polymers' must be limited to purely organic polymers, since we find included three papers dealing with inorganic polymers. There are twenty-two papers in all and, with the vast range of topics available, it is scarcely surprising that they are as heterogeneous as some of the materials they describe. Although the majority of authors are British, there are also contributions from continental Europe, the United States and Australia. Generally speaking, this is not a book for the theoretician, but rather for the practical scient:st who wants to know how to make things, how and where to apply them, what their important properties are, and how to measure them.

Topics are divided roughly into six sections : preparation of non-oxides; pyrolytics and inorganio polymors; oxides; proporties; fabrication techniques; and applications. The first section has one paper on the preparation of new hightemperature materials for atomic energy usage, another in which modorn theory is applied to an old sintering problem, and the third is a structure analysis of zirconium oxycarbides. The first two of the second group of papers deal with the technologically very interesting formation of hard, impervious, inert layers of silicon carbide on various substrates. The second paper, on inorganic polymers, contains much introductory and background information and, I feel, could well have come first in the section. The other two deal with the preparation of specific polymers. The four papers in the oxide group consist of one on the preparation of high-purity powders, another on the problem of alumina analysis, a third on the improvement of high-temperature structural material by metal infiltration, 